\title{
Numerical Research on Flow Characteristics around a Hydraulic Turbine Runner at Small Opening of Cylindrical Valve
}

\author{
Zhenwei Mo, Juliang Xiao, and Gang Wang \\ Key Laboratory of Mechanism Theory and Equipment Design of Ministry of Education, Tianjin University, Tianjin 300072, China \\ Correspondence should be addressed to Juliang Xiao; tianjinxjl@163.com
}

Received 14 September 2015; Revised 25 November 2015; Accepted 25 November 2015

Academic Editor: David Bigaud

Copyright ( 2016 Zhenwei Mo et al. This is an open access article distributed under the Creative Commons Attribution License, which permits unrestricted use, distribution, and reproduction in any medium, provided the original work is properly cited.

\begin{abstract}
We use the continuity equation and the Reynolds averaged Navier-Stokes equations to study the flow-pattern characteristics around a turbine runner for the small-opening cylindrical valve of a hydraulic turbine. For closure, we adopt the renormalization-group $k-\varepsilon$ two-equation turbulence model and use the computational fluid dynamics (CFD) software FLUENT to numerically simulate the three-dimensional unsteady turbulent flow through the entire passage of the hydraulic turbine. The results show that a lowpressure zone develops around the runner blades when the cylindrical valve is closed in a small opening; cavitation occurs at the blades, and a vortex appears at the outlet of the runner. As the cylindrical valve is gradually closed, the flow velocity over the runner area increases, and the pressure gradient becomes more significant as the discharge decreases. In addition, the fluid flow velocity is relatively high between the lower end of the cylindrical valve and the base, so that a high-velocity jet is easily induced. The calculation and analysis provide a theoretical basis for improving the performance of cylindrical-valve operating systems.
\end{abstract}

\section{Introduction}

Cylindrical valve installed between the stay and guide vane of mixed-flow hydraulic turbine boasts many advantages such as self-closing ability, less hydraulic loss, effective protection to the distributor, small land occupation in factory, and small investment. So cylindrical valve has been widely used in large- and medium-sized hydropower stations over sediment-laden rivers. For large hydropower stations involving peak load regulation and frequency modulation where conventional inlet valve (ball valve and butterfly valve) cannot be installed, the installation of cylindrical valve can effectively protect the distributor and reduce the leakage loss in the stopping process. The world's first cylindrical valve was made by NEYRPIC Company in France for Monteynard Power Station in 1962. The power station had an installed capacity of $4 \times 83 \mathrm{MW}$ and a water head of $137 \mathrm{~m}$. So far, cylindrical valve has been used in over 60 power units in 12 hydropower stations in France, Canada, Portugal, and so forth. In China, Xiaowan Hydropower Station has 6 Francis turbine generator units with a unit capacity of $700 \mathrm{MW}$ and Nuozhadu Hydropower Station has 9 hydraulic turbine generator units with a unit capacity of $650 \mathrm{MW}$ [1].
A runaway transient in a hydraulic turbine is an abnormal process that adjusts the hydraulic turbine; therefore an indepth understanding of such transients is vital for the safe and normal operation of hydraulic turbines. When a cylindrical valve is used to shut down the turbine by forcing the hydraulic turbine to exit the runaway state, the water flow surrounding the runner of the hydraulic turbine is extremely turbulent and accompanied by strong water impact, vibration, and noise. This is especially true when the cylindrical valve is closed in a small opening. All of these unstable phenomena can lead to disastrous accidents involving the hydraulic turbine unit [2-6]. Therefore, for proper design of a hydropower station, a vital prerequisite is the calculation, analysis, and forecast of the flow field surrounding the runner when the hydraulic turbine exit the runaway state. Such research provides important parameter values that allow the hydraulic turbine to exit the runaway process in a fast, safe, and stable manner.

When the cylindrical valve is used to shut down the turbine, the flow field becomes extremely turbulent. Experiments modeling this regime are of limited use because the methods used to interpolate between the model turbine and the prototype turbine are not perfect. Shutting down a real 
hydraulic turbine in the runaway state involves a significant risk, which precludes performing such experiments with a real hydraulic turbine. Thus, we adopt a numerical approach in this study, in which we use computational fluid dynamics (CFD) to analyze and study the process of shutting down a turbine with a cylindrical valve.

In recent years, many research groups in China have numerically simulated hydraulic turbines. A group at the State Key Laboratory of Hydroscience and Engineering of Tsinghua University [7-9] studied the rotation rate, hydrodynamic moment, and the characteristic runaway curve for runaway transients in Francis turbines. These studies led to a thorough understanding of the flow through the entire passage of the hydraulic turbine and provided an analysis of the vortex and pressure fluctuations. In another work, $\mathrm{Li}$ et al. [10] considered the use of a cylindrical valve for the emergency shutdown of a turbine device and analyzed and compared the relevant parameters of cylindrical valves. These experiments and the related numerical analysis were carried out to understand how the shape of the cylindrical valve's lower end influences the surrounding flow field. Wührer and Grein [11] calculated and analyzed the hydrodynamic force at the cylindrical valve of a hydraulic turbine for a noncontinuous flow. A group at Tianjin University [12-15] carried out a three-dimensional (3D) numerical analysis of the entire passage of a hydraulic turbine, studied a multicylinder coordinated control strategy destined to shut down the turbine with the help of a cylindrical valve, comparatively analyzed analysis of several different shutdown methods involving cylindrical valves with different bottom shapes, and found that the hydraulic characteristics are well in the manner of the $60 \mathrm{~s}$ closing.

However, these studies did not analyze the flow pattern around the turbine runner when the cylindrical valve is enclosed in a small opening. Thus, we address this issue herein by performing numerical CFD simulations of a prototype cylindrical valve for the Nansha hydropower station in Yunnan, China. We focus on analyzing the flow field around the turbine runner with the cylindrical valve at a small opening.

\section{Description of the Simulation}

Figure 1 shows a schematic of the entire flow passage and cylindrical valve of the prototype hydraulic turbine of the Nansha hydropower station. The photograph of the cylindrical valve with a unit capacity of $50 \mathrm{MW}$ in Nansha Hydropower Stations of Yunan, China, is shown in Figure 2. The degree $e$ of the relative opening of the cylindrical valve determines how quickly the turbine is shut down and is defined as the ratio of the distance $d$ between the cylindrical valve's lower end and the bottom ring to the thickness of the valve $D: e=d / D$. The cylindrical valve is $110 \mathrm{~mm}$ thick and $1405 \mathrm{~mm}$ high. According to the definition of the degree of relative opening, the cylindrical valve must limit $e$ to between 0 and 12.7 during turbine shutdown. For a small opening, $0<e<1$. The angle $\varphi$ is the nose angle, as shown in Figure 3. We acquired screenshots of the cross section for $\varphi=90^{\circ}$ to $270^{\circ}$ and $\varphi=0$ to $180^{\circ}$. Similarly, pictures of the lower end

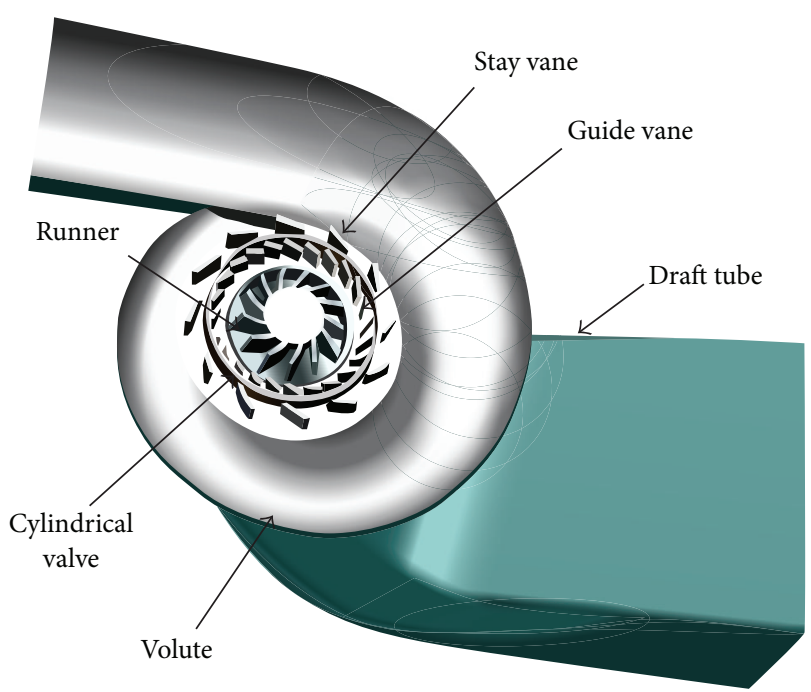

FIGURE 1: Schematic of the model components of the turbine and a photograph of the cylindrical valve.

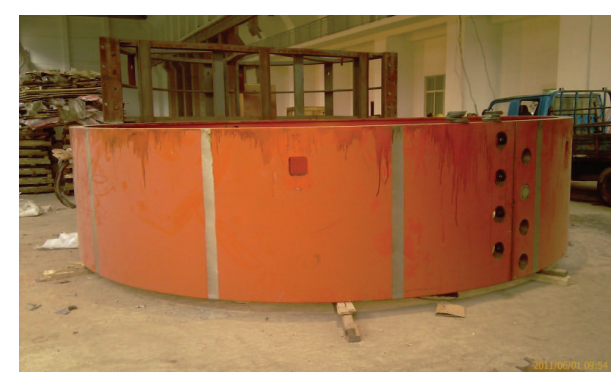

FIGURE 2: Photograph of the cylindrical valve.

of the cylindrical valve with nose angles of $0^{\circ}, 90^{\circ}, 180^{\circ}$, and $270^{\circ}$ are also available.

\section{Numerical Calculation}

3.1. Control Equation. When the cylindrical valve is closed for emergency shutdown of the turbine, the fluid flows inside the turbine and around the cylindrical valve become a complex, 3D unsteady incompressible turbulent flows. Because the renormalization-group $k-\varepsilon$ turbulence model distinguishes between a flow and a swirling flow, it can predict the flow pattern near a wall surface relatively well. In fact, this approach has been used in many studies to simulate $3 \mathrm{D}$ flow in fluid machinery [16]. Thus, we also adopt this turbulence model for the present study. The flow-control equations used herein for the cylindrical-valve region are the continuity equation

$$
\frac{\partial u_{i}}{\partial x_{i}}=0
$$

the momentum equation

$$
\frac{D u_{i}}{D t}=f_{i}-\frac{1}{\rho} \frac{\partial p}{\partial x_{i}}+\frac{\partial}{\partial x_{j}}\left(\nu \frac{\partial u_{i}}{\partial x_{j}}-\overline{u_{i}^{\prime} u_{j}^{\prime}}\right),
$$




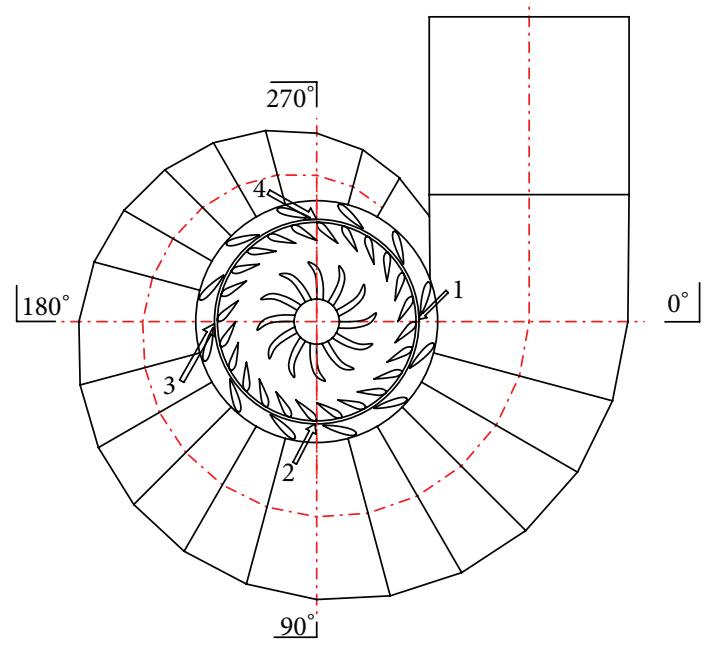

FIGURE 3: Schematic of the hydraulic turbine showing the nose angle.

and the renormalization-group $k-\varepsilon$ equation

$$
\begin{aligned}
& \frac{D k}{D t}=\frac{\partial}{\partial x_{i}}\left[\left(v+\frac{v_{t}}{\sigma_{k}}\right) \frac{\partial k}{\partial x_{i}}\right]+G_{k}-\varepsilon, \\
& \frac{D \varepsilon}{D t}=\frac{\partial}{\partial x_{i}}\left[\left(v+\frac{v_{t}}{\sigma_{\varepsilon}}\right) \frac{\partial \varepsilon}{\partial x_{i}}\right]+C_{1 \varepsilon} \frac{\varepsilon}{k} G_{k}-C_{2 \varepsilon} \frac{\varepsilon^{2}}{k},
\end{aligned}
$$

where $x_{i}(i=1,2,3)$ are the Cartesian coordinates, $u_{i}$ $(i=1,2,3)$ are the velocity components, $p$ is the corrected pressure, $v$ is the kinematic viscosity coefficient, $f_{i}$ is the mass force, and $\nu_{t}$ is the turbulent viscosity coefficient $\left(v_{t}=\right.$ $\left.c_{u} k^{2} / \varepsilon\right), c_{u}=0.09, \sigma_{k}=1.0, \sigma_{\varepsilon}=1.33, C_{1 \varepsilon}=1.44$, and $C_{2 \varepsilon}=1.42$. Because the Reynolds stress $-\overline{u_{i} u_{j}}$ in (2) is problematic for closure, the renormalization group $k-\varepsilon$ twoequation model is used for closure [17].

Since the cylindrical valve of hydraulic turbine is in a state of motion during emergency shutdown process, which can cause change in the calculation area of the flow field, dynamic mesh technique was used to adapt to this change. This study divided the motion process of cylindrical valve into 48 time steps, each time step corresponding to a different displacement of the cylindrical valve. Firstly, the torque $M$ of the water flow action on the blade of hydraulic turbine on the first time step was calculated. Then the angular velocity increment of the blade of hydraulic turbine in each time step was obtained according to (4), and the incremental rotational speed of the hydraulic turbine was further calculated. In this way, the rotational speed of hydraulic turbine on the first time step can be obtained according to its initial rotational speed. In the same manner, the rotational speed on each time step can be obtained:

$$
M-M_{f}=J \Delta \omega,
$$

where $M_{f}$ is the resistance moment; $J$ is the rotational inertia of the blade of hydraulic turbine; $\Delta \omega$ is the angular velocity increment on each time step.
Since the runner is a rotating component, rotor-stator interaction will occur between cylindrical valve and runner flow fields and between draft tube and runner flow fields. In order to simulate detailed changes of the flow field over time, sliding mesh was used in the front of the runner inlet and the back of the runner outlet (namely, the interface of adjacent subdomains) to complete the data transmission for the entire computational domain.

3.2. Mesh and Boundary Conditions. For the numerical simulation, we created a 1:1 model of a cylindrical valve in the 3D modeling software UG. The computational control field is the entire passage from the entrance of the hydraulic turbine's spiral casing to the exit of the draft tube. Note that the creation of the mesh for the flow passage of the hydraulic turbine is an essential part of the entire numerical calculation process because the speed, accuracy, and convergence of the flow-field calculation for the flow passage of the hydraulic turbine are directly related to the quality of the mesh. For the present work, we use ICEM CFD as the preprocessing module. Considering the specific situations and requirements for this work, the entire passage of the virtual hydraulic turbine was divided into six regions. A structured grid was used for the region of the cylindrical valve that involves motion; unstructured grids were used for other regions. The grid close to the cylindrical valve was densified to improve the calculation accuracy of the surrounding flow field. The final number of computational grid elements was $3 \times 10^{8}$. The resulting meshes for the hydraulic turbine runner and cylindrical valve are shown in Figure 4.

Because the numerical simulation covers only a limited computational domain, specific boundary conditions must be applied at each boundary crossed by the flow in the hydraulic turbine. The boundary conditions for the turbine's internal flow field mainly involve the inlet, outlet, and wall boundary conditions. Experience from the Nansha hydropower station indicates that a cylindrical valve is used to shut down the turbine when the water flow cannot be cut off in time (owing to the runaway phenomenon in the turbine or the breakdown of the guide-vane apparatus). Therefore, the boundary conditions should be applied to the inlet, outlet, and wall of the entire flow passage through the spiral casing. The outlet pressure of the draft-tube outlet gives the outlet pressure condition, and the inlet pressure of the spiral casing gives the inlet pressure condition. On the basis of real operating conditions of the Nansha hydropower station, we use a $50 \mathrm{~m}$ head at the spiral casing's inlet as the inflow boundary condition with an initial flow speed of $5.13 \mathrm{~m} / \mathrm{s}$. Because the outlet of the draft tube is directly connected to nearby rivers, the outlet is assumed to be at standard atmospheric pressure. However, the effect of gravity on the pressure distribution is taken into consideration because of the considerable height of the draft tube of the turbine.

For the simulation, we used the FLUENT software package. Numerically calculation of the initial flow field of the hydraulic turbine is a transient problem; therefore, the first step is to calculate the initial steady-state flow field, and the result of this calculation is used as the initial condition for the next step. The finite-volume method (a common 


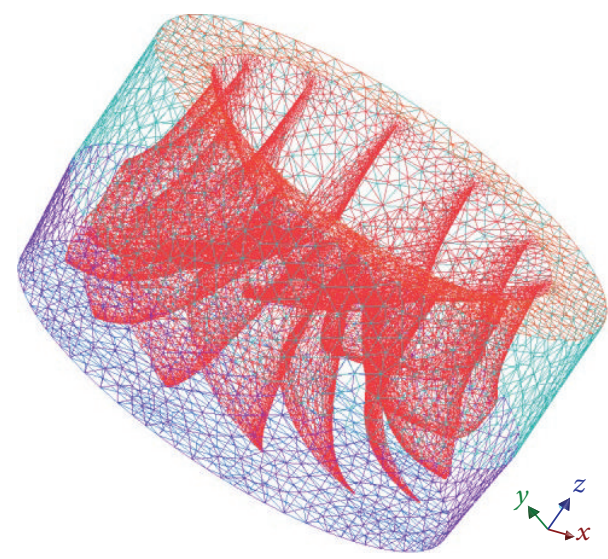

(a)

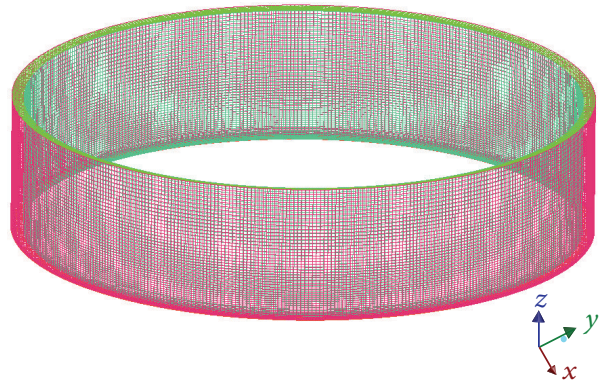

(b)

Figure 4: Mesh for the (a) hydraulic turbine runner and (b) cylindrical valve.

CFD numerical method) is used to discretize the control equations. We use the second-order upwind scheme to generate the convection term and the second-order centraldifference scheme to generate the diffusion and pressure terms of the motion equation. To iterate the flow field in the hydraulic turbine, we use the semi-implicit algorithm for pressure-coupled equations to solve the problem of velocity and pressure coupling in the incompressible Navier-Stokes equations [14]. This algorithm is widely used to calculate the flow field in fluid machinery and often used to calculate the flow field in hydraulic turbines.

3.3. Model Validation. The numerical model was verified by testing if its results are independent of the grid via a comparison of results obtained with three different grid systems with approximately $1.2 \times 10^{8}, 1.8 \times 10^{8}$, and $3.0 \times$ $10^{8}$ grid elements, which describe the entire passage of the hydraulic turbine grid elements. In addition, the grids are densified near the cylindrical valve because the fluid field must be accurately represented in this area. The calculation results for the $1.2 \times 10^{8}$ and $3.0 \times 10^{8}$ element grid systems differ by less than $5 \%$; therefore, we use the latter for the calculation and analysis presented herein. Figure 5 shows a comparison of the downpull force with real machine test results [18]. From Figure 5, it can be seen that the downpull force predicted by the numerical method is in substantial agreement with the real machine test results.

\section{Analysis of the Results}

4.1. Analysis of the Flow Pattern of the Runner Area. The runner is the crucial factor for determining the internal flow field of the hydraulic turbine during shutdown by the cylindrical valve. Thus, a proper understanding of the hydraulic characteristics of the runner area is vital. Because the rotating runner affects the direction and magnitude of the internal flow field of the cylindrical valve, these quantities are difficult to determine with precision. As a result, the flow pattern created by the cylindrical valve in the runner area is

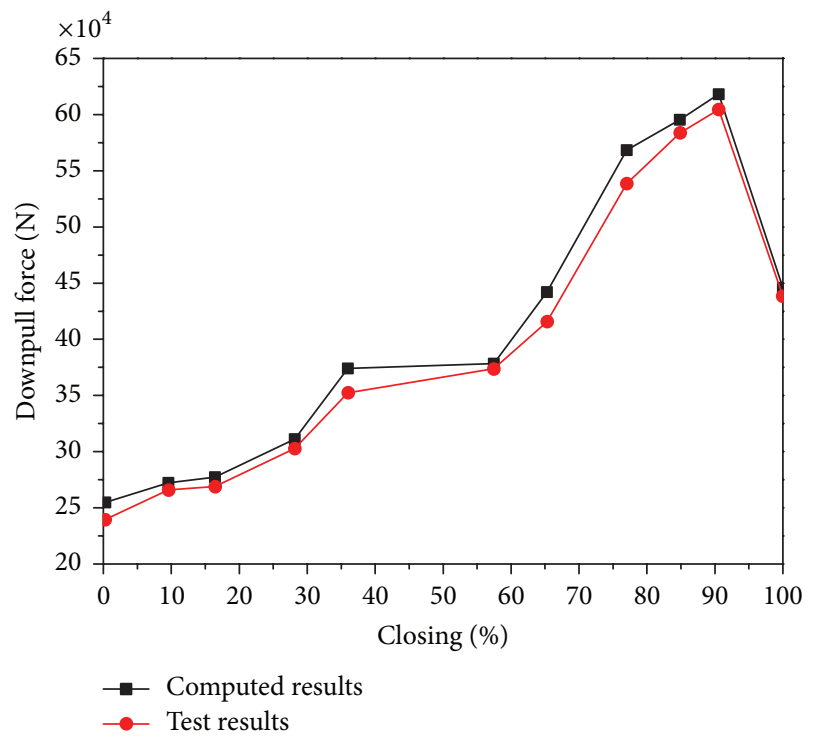

FIGURE 5: Downpull force compared with real machine test results.

extremely turbulent. In the present work, we compare and analyze the pressure and velocity fields of a cylindrical valve with $e=0.3$ and 0.9 .

As shown in Figures 6 and 7, the pressure is unevenly distributed over the turbine blades. Specifically, the bladeinlet area experiences the highest pressures, whereas the blade-outlet blade area experiences the lowest pressure. These results are consistent with the fact that the runner converts the translational kinetic energy of the water flow into the rotational kinetic energy of the turbine. Furthermore, the small pressure gradient at the outlet satisfies the outlet pressure-gradient requirements. When the runner rotates, the water flow rotating at a high speed generates large centrifugal forces, thereby generating an external pressure that exceeds the internal pressure. With the decrease in the pressure of the flowing fluid, the pressure on the impact side of the blades far exceeds that on the back side of the blades. As 


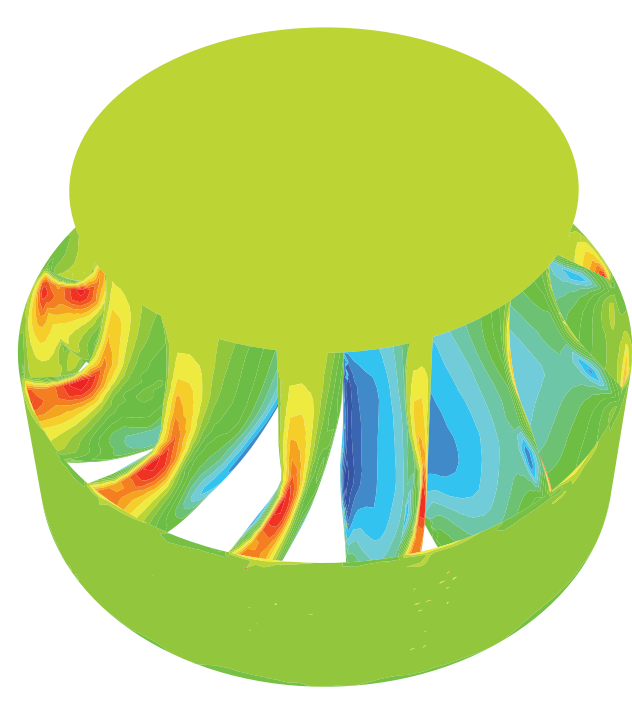

(a)

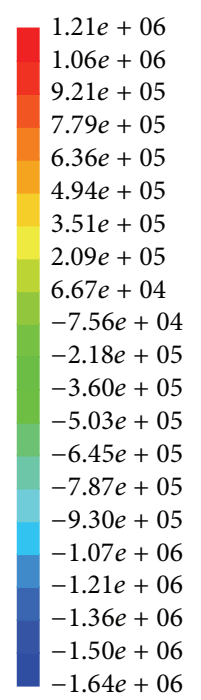

$-1.64 e+06$

FIGURE 6: Pressure distributions of a runner observed from the isometric (a) and bottom (b) views. The degree of the relative opening of the cylindrical valve is 0.3 .

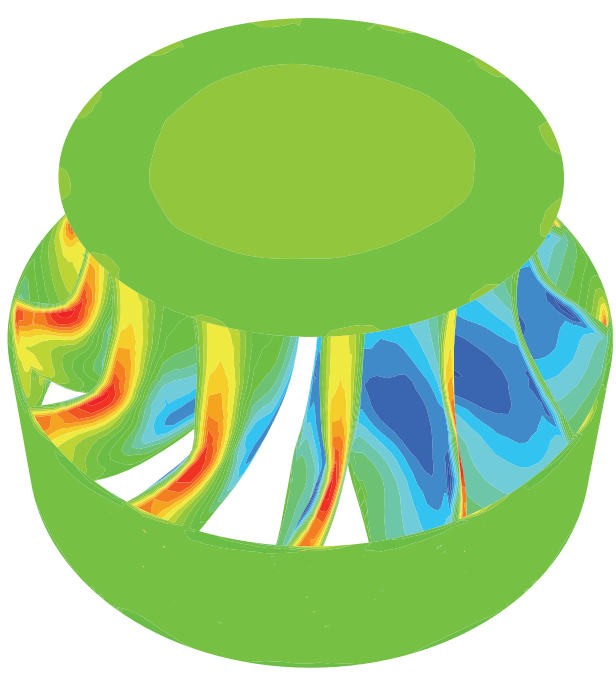

(a)

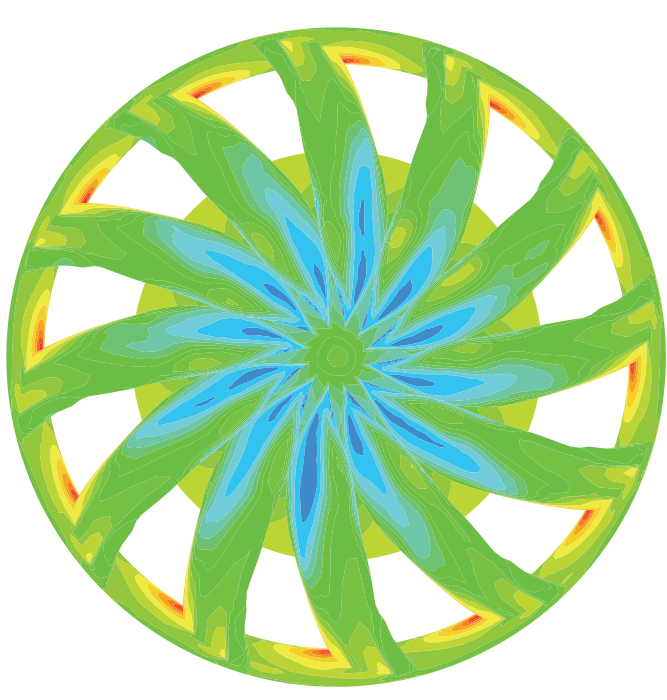

(b)
$1.21 e+06$

$1.06 e+06$

$9.21 e+05$

$7.79 e+05$

$6.36 e+05$

$4.94 e+05$

$3.51 e+05$

$2.09 e+05$

$6.67 e+04$

$-7.56 e+04$

$-2.18 e+05$

$-3.60 e+05$

$-5.03 e+05$

$-6.45 e+05$

$-7.87 e+05$

$-9.30 e+05$

$-1.07 e+06$

$-1.21 e+06$

$-1.36 e+06$

$-1.50 e+06$

$-1.64 e+06$

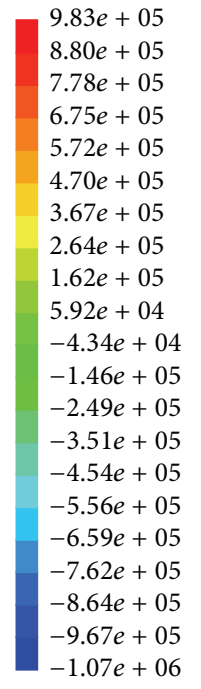

$-1.07 e+06$

FIGURE 7: Pressure distributions of a runner observed from the isometric (a) and bottom (b) views. The degree of the relative opening of the cylindrical valve is 0.9 .

shown in Figures 6(b) and 7(b), a low-pressure zone develops on the back side of the blades, creating a negative pressure. Such low-pressure zones are propitious for the cavitation phenomenon.

As shown in Figures 8 and 9, the flow velocity in the runner area increases when the cylindrical valve is closed, and the degree of relative opening decreases. In addition, the pressure gradient in the runner area becomes more apparent as the flux decreases. When the runner rotates at high speeds, a secondary flow is likely to form in the runner area. As shown in the plane sketch of the runner blades (Figures 8(a) and $9(\mathrm{a}))$, the secondary flow lines are seriously distorted, and the flow pattern is extremely turbulent. Owing to the impact created by the large angle of attack at the inlet, a channel

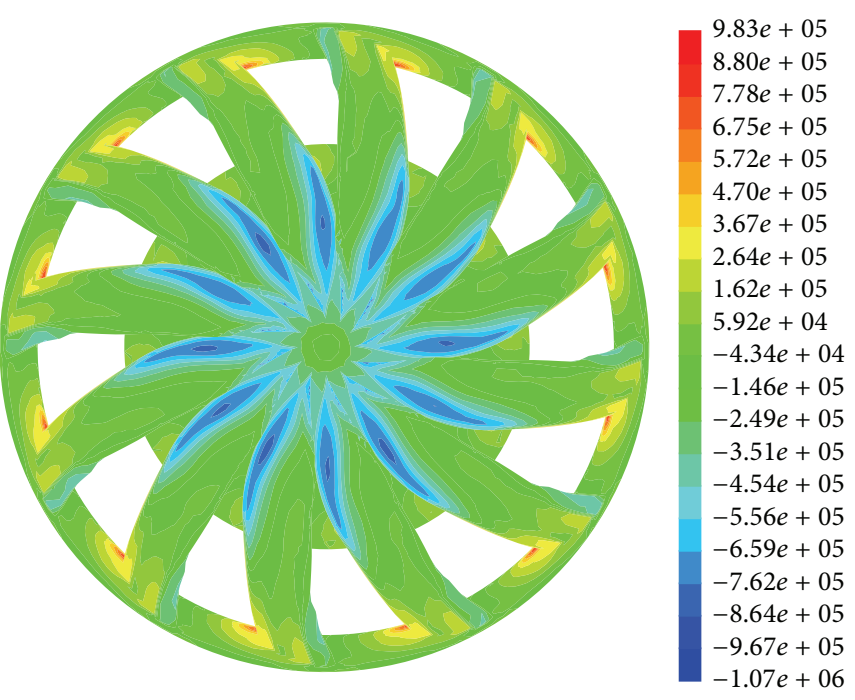

(b) vortex forms between the blades because of the secondary flow and backflow between the flow passages. As shown in the water-flow vector diagram of the runner outlet (Figure 9), a huge swirling flow forms at the runner outlet with a high outer speed and low inner speed, and these angular velocities increase as the degree of the relative opening of the cylindrical valve decreases. Because the vortex is off center, we deduce that a larger off-center vortex should form at the turbine's draft-tube inlet because of the transfer of water. The channel vortex and the runner vortex exert a significant stress on the runner blades, which shifts the blades, thereby intensifying blade cavitation and pressure pulsation. As the amplitude of the blade vibration increases, spots, cracks, and even spongelike damage may appear on the surfaces of the runner blades. 


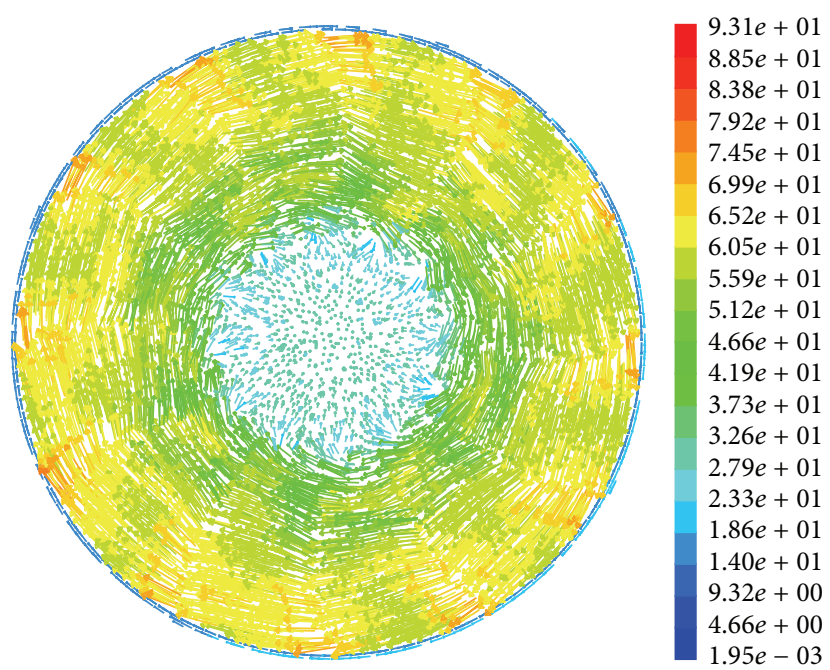

(a)

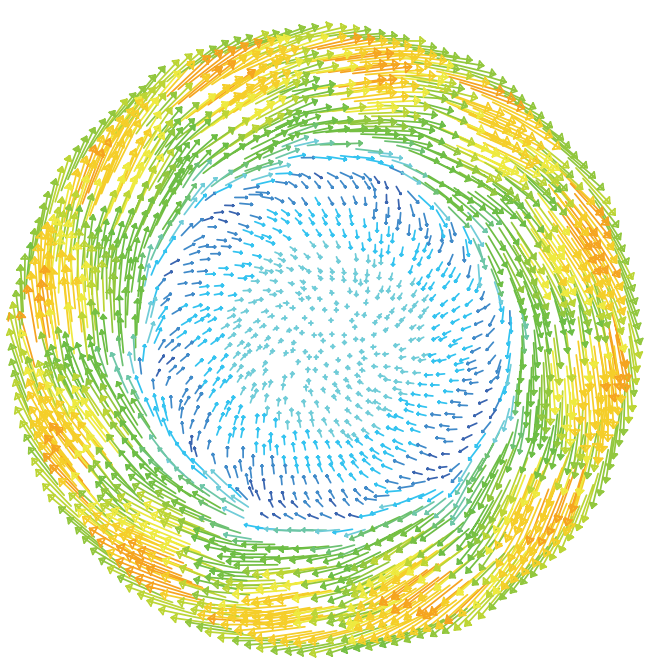

(b)
$7.00 e+01$

$6.65 e+01$

$6.30 e+01$

$5.95 e+01$

$5.60 e+01$

$5.25 e+01$

$4.90 e+01$

$4.55 e+01$

$4.20 e+01$

$3.85 e+01$

$3.50 e+01$

$3.15 e+01$

$2.80 e+01$

$2.45 e+01$

$2.10 e+01$

$1.75 e+01$

$1.40 e+01$

$1.05 e+01$

$7.00 e+00$

$3.50 e+00$

$1.95 e-03$

FIGURE 8: Velocity vector diagram of a runner in the $x-y$ plane and at the outlet when the degree of the relative opening of the cylindrical valve is 0.3 .

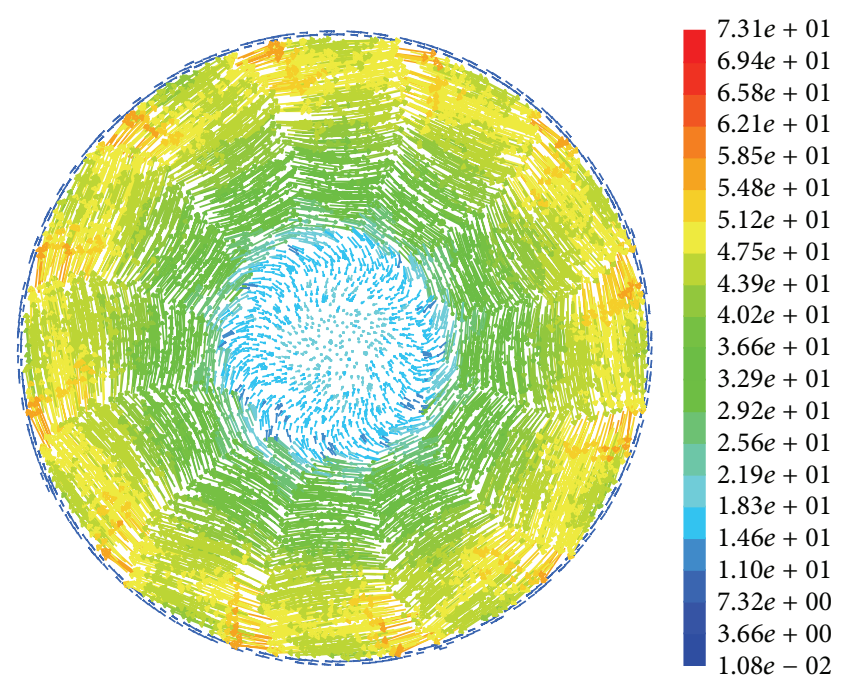

(a)

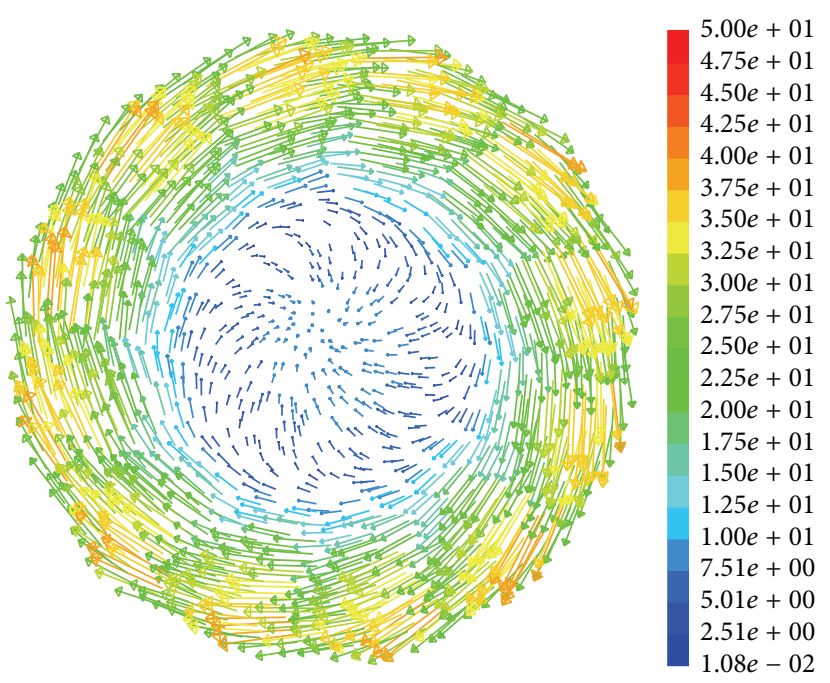

(b)

FIGURE 9: Velocity vector diagram of a runner in the $x-y$ plane and at the outlet when the degree of the relative opening of the cylindrical valve is 0.9 .

4.2. Distribution of the Flow Field around a Cylindrical Valve. Because the water flow near the lower end of the cylindrical valve is turbulent, we amplify this area to analyze the pressure and velocity fields. Cross-sectional views for nose angles of $0^{\circ}$, $90^{\circ}, 180^{\circ}$, and $270^{\circ}$ are shown in Figures 10 and 11 for $e=0.3$ and 0.9 , respectively.

As shown in Figures 10 and 11, the inner and outer pressures greatly differ at and next to the throttling point, which is between the lower end of the cylindrical valve and the bottom ring. This creates a pressure gradient along the intervening path. This result indicates that a considerable hydraulic loss occurs at the lower end of the cylindrical valve when enclosed in a small opening. Furthermore, a jet-prone condition is created because of the small degree of opening and the large flow velocity. Consequently, the pressure on the cylindrical valve should decrease accordingly.

A low-pressure zone, which apparently forms at the outlet of the cut-off section of the cylindrical valve, is a bubblegenerating area. The inner surface of the valve is likely to be impacted by the local water flow, thereby generating cavitation noise and vibration. The bursting bubbles in this manner would lead to the long run to corrosion of the valve surface over the long run further amplifying cavitation and cavitation erosion.

\section{Conclusion}

In this work, we calculated and analyzed the flow field around the runner of a small-opening cylindrical valve for a turbine. 


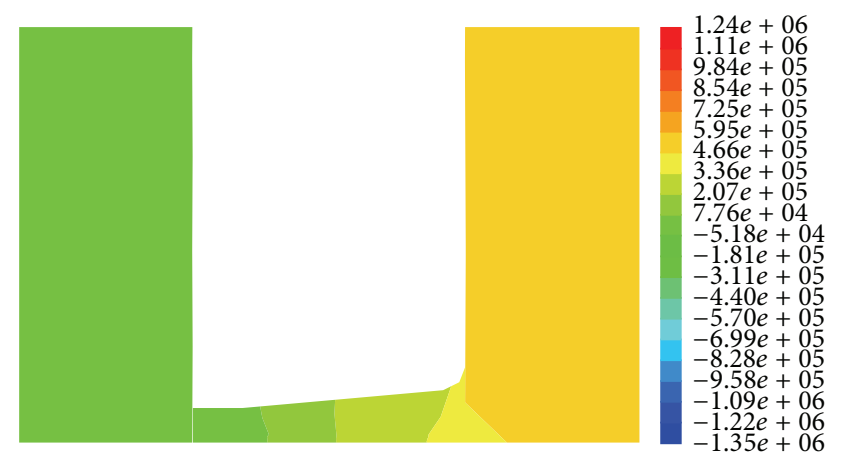

(a) $\varphi=0^{\circ}$

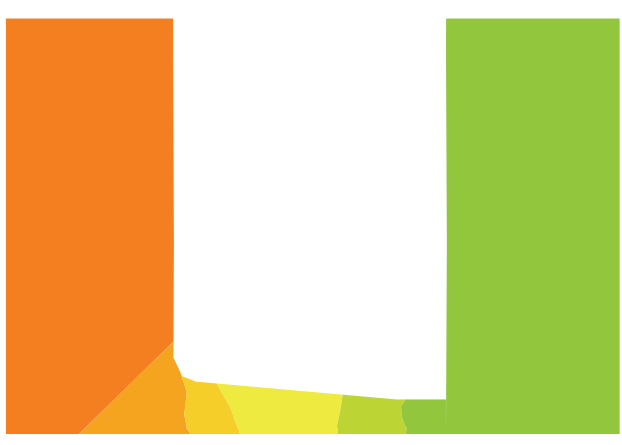

(c) $\varphi=180^{\circ}$

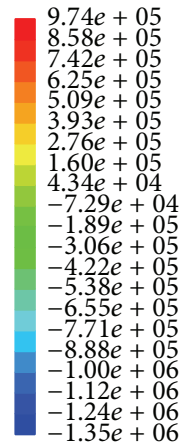

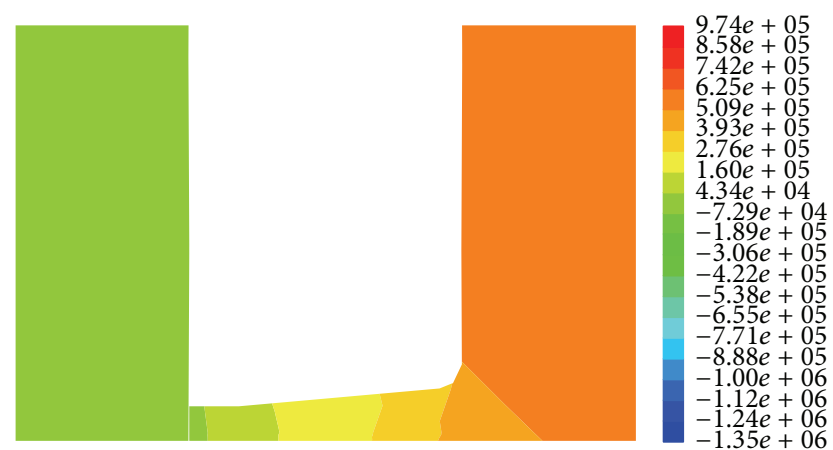

(b) $\varphi=90^{\circ}$
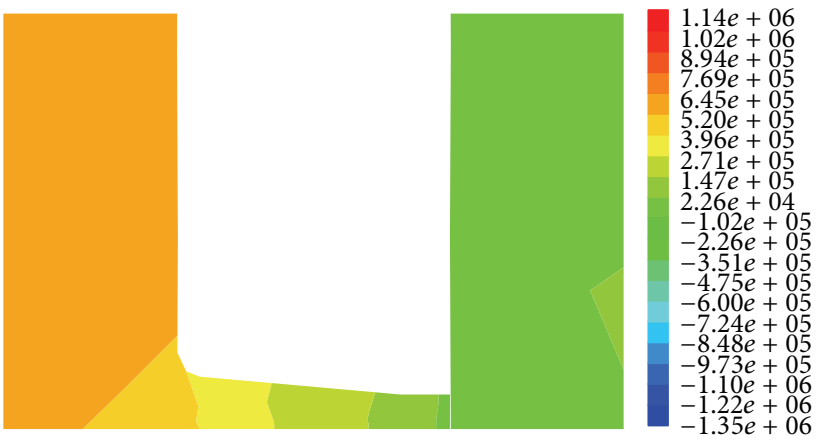

(d) $\varphi=270^{\circ}$

FIgURe 10: Pressure distributions around a cylindrical valve for various nose angles $\varphi$ and $e=0.3$.

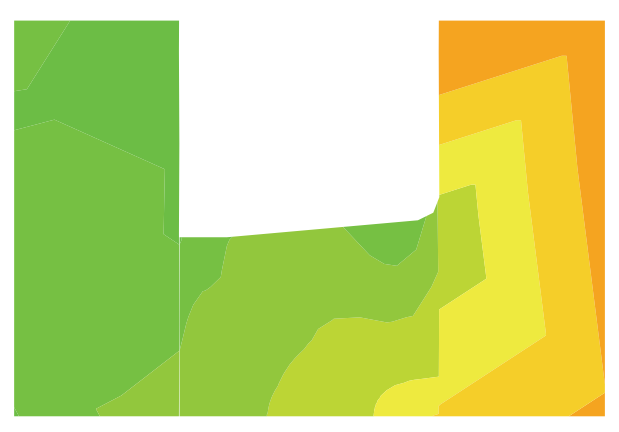

(a) $\varphi=0^{\circ}$

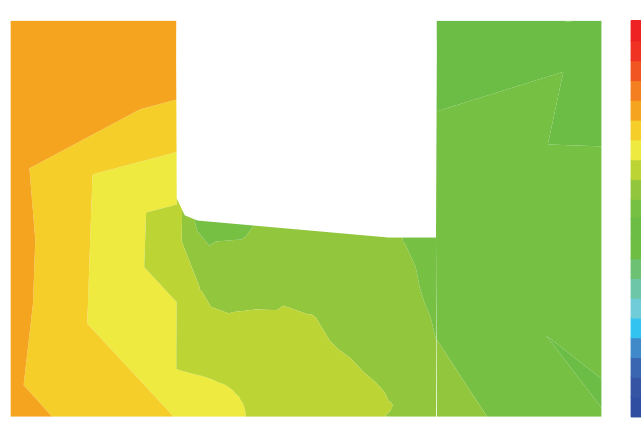

(c) $\varphi=180^{\circ}$
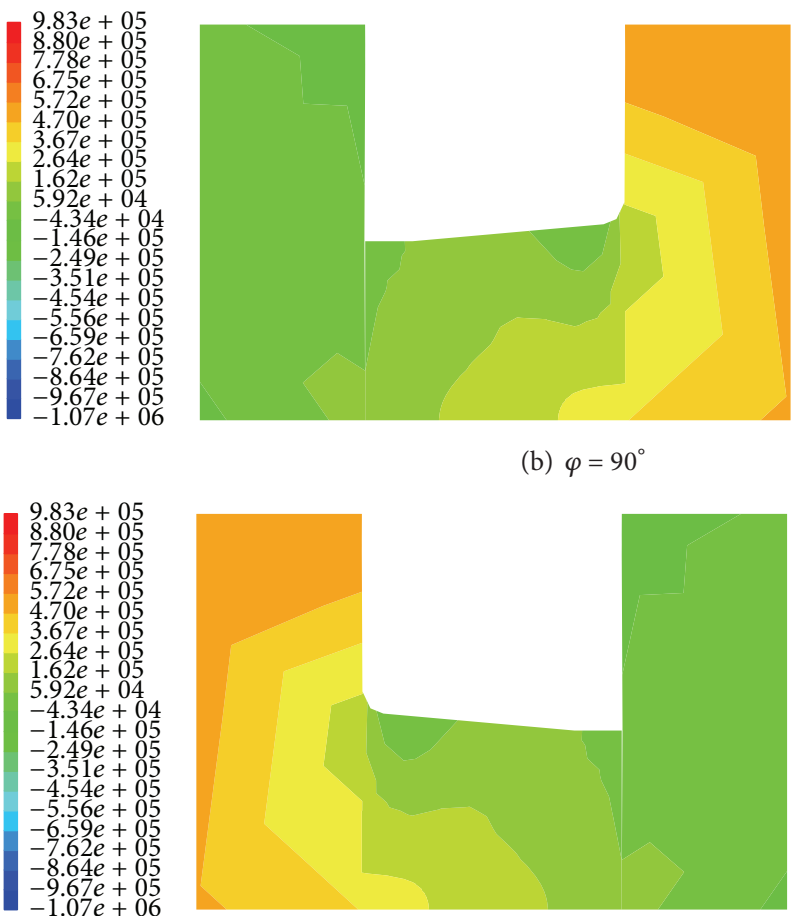

(b) $\varphi=90^{\circ}$

(d) $\varphi=270^{\circ}$
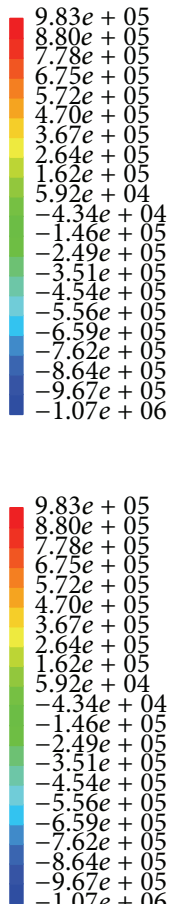

$-9.67 e+05$
$-1.07 e+06$

(d) $\varphi$

FIGURE 11: Pressure distributions around a cylindrical valve for various nose angles $\varphi$ and $e=0.9$. 
By analyzing the pressure and velocity fields of such a valve, we find that a low-pressure zone develops on the runner blades, cavitation erosion occurs on the blades, and a vortex forms at the runner outlet. The velocity distribution map of the runner area shows that the flow velocity near the runner increases upon closing the cylindrical valve. In addition, the pressure gradient increases as the flux decreases. When the runner rapidly rotates, secondary water is likely to flow through the runner area. As shown in the plane sketches of the runner blades, the secondary-flow line is seriously distorted and the flow pattern is extremely turbulent. The inner and outer pressures greatly vary at and next to the throttling point, which is between the lower end of the cylindrical valve and the bottom ring, revealing a pressure gradient along the intervening path. This result indicates that a considerable hydraulic loss occurs at the lower end of the small-opening cylindrical valve. In addition, a jetprone condition occurs because of the small degree of relative opening and the large flow velocity.

\section{Conflict of Interests}

The authors declare no conflict of interests regarding the publication of this paper.

\section{Acknowledgments}

This work was sponsored by the Key Technologies R\&D Program of Tianjin under Grant no. 09ZCKFGX03400 and by the Youth Scholar Foundation (B type) of Tianjin University under Grant no. TJU-YFF-08B24.

\section{References}

[1] S. B. Wu, "Research on cylindrical valve at Nuozhadu hydropower plant," Mechanical \& Electrical Technique of Hydropower Station, vol. 31, no. 2, pp. 13-15, 2008.

[2] Z. Q. Tian and J. W. Liu, "Study on hydraulic stability of francis turbine of Three Gorges Power Station," Yangtze River, vol. 31, no. 5, pp. 1-3, 2000.

[3] M. Q. Ji and J. L. Yuan, "Turbine and its characteristics," Heilongjiang Science and Technology of Water Conservancy, vol. 4, pp. 134-134, 2009.

[4] Y. D. Zhao, H. J. Hong, and Y. Li, "Discussion on anti-runaway design for units of Binling hydropower station," Northwest Hydropower, vol. 1, pp. 47-51, 2009.

[5] G. Q. Ma, H. J. Duan, G. F. Liu et al., "Analysis of cylinder valve installation at Wunonglong hydropower station," Northwest Hydropower, vol. 3, pp. 69-72, 2011.

[6] Z. Q. Wang, "Simple explanation on runaway protection of hydro-generator unit," Hubei Water Power, vol. 6, pp. 72-73, 2011.

[7] W. R. Strub and R. E. Mawhinney, "Ring gates for La grande-2 turbines," Water Power, vol. 31, pp. 11-13, 1979.

[8] Y. L. Wu, S. H. Liu, and Z. D. Qian, Shuilijixie Jisuan Liuti Donglixue, Water \& Power Press, Beijing, China, 2007.

[9] S. H. Liu, L. Zhang, Y. L. Wu, X. W. Luo, and M. Nishi, "Influence of $3 \mathrm{D}$ guide vanes on the channel vortices in the runner of a Francis turbine," Journal of Fluid Science and Technology, vol. 1, no. 2, pp. 147-156, 2006.
[10] J. W. Li, S. H. Liu, D. Q. Zhou, and Y. L. Wu, "3D unsteady turblulent simulation of the runaway transients of the francis turbine," Journal of Hydroelectric Engineering, vol. 27, no. 6, pp. 148-152, 2008.

[11] W. J. Wührer and H. L. Grein, "Ring gate as turbine emergency shut-down device," Hydraulic Engineering, vol. 6, pp. 1085-1090, 1990.

[12] I. S. Veremeenko and S. D. Kostornoy, "Computation of hydrodynamic forces at the ring gate of a hydraulic turbine," Tyazheloe Mashinostroenie, vol. 10, pp. 5-7, 1992.

[13] C. L. Guo, J. L. Xiao, and G. D. Wang, "Numerical simulation for characteristics of ring gate during emergency shut-down water process," Journal of Tianjin University, vol. 42, no. 11, pp. 10221027, 2009.

[14] M. Li, J. L. Xiao, G. Wang, and W. K. Song, "Three-dimensional unsteady numerical simulation of ring gate emergency shutdown for hydraulic turbine," Journal of Hydroelectric Engineering, vol. 31, no. 2, pp. 228-234, 2012.

[15] J. Xiao, E. Zhu, and G. Wang, "Numerical simulation of emergency shutdown process of ring gate in hydraulic turbine runaway," Journal of Fluids Engineering, vol. 134, no. 12, Article ID 124501, 2012.

[16] C. L. Guo, G. D. Wang, and J. L. Xiao, "Numerical simulation for hydraulic characteristics of cylindrical valve in runaway protection process," in Proceedings of the Asia-Pacific Power and Energy Engineering Conference, Wuhan, China, March 2009.

[17] J.-F. Huang, L.-X. Zhang, and S.-H. He, "Numerical simulation of 3-D steady and unsteady flows in whole flow passage of a francis hydro-turbine," Proceedings of the Chinese Society of Electrical Engineering, vol. 29, no. 2, pp. 87-94, 2009.

[18] Z. W. Mo, J. L. Xiao, and G. Wang, "Evaluation of hydrodynamic forces on turbine ring gate during emergency shutdown process," Advances in Mechanical Engineering, vol. 7, no. 5, pp. 1-10, 2015. 


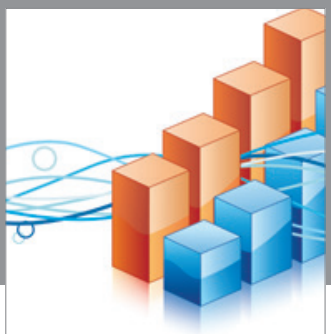

Advances in

Operations Research

vatem alat4

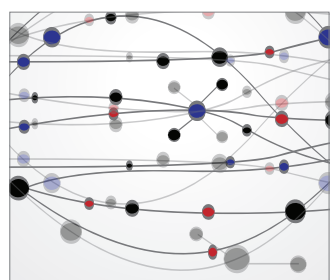

\section{The Scientific} World Journal
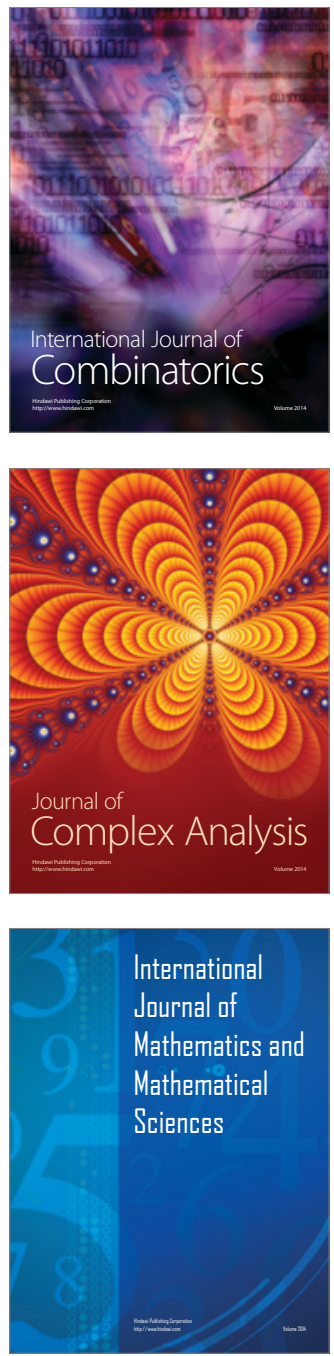
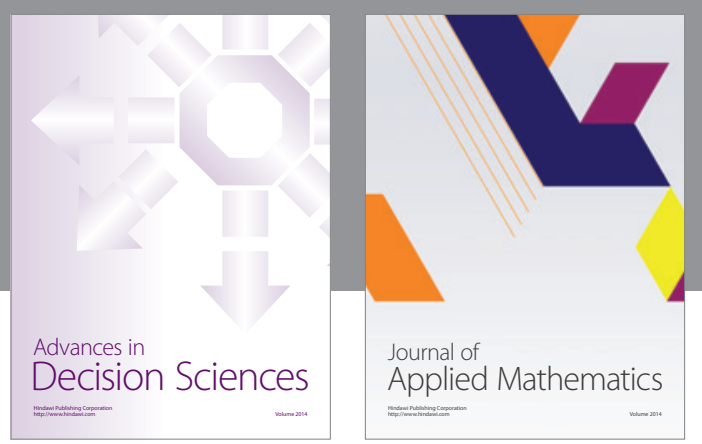

Algebra

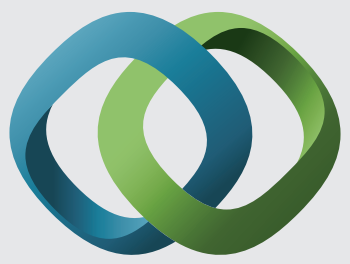

\section{Hindawi}

Submit your manuscripts at

http://www.hindawi.com
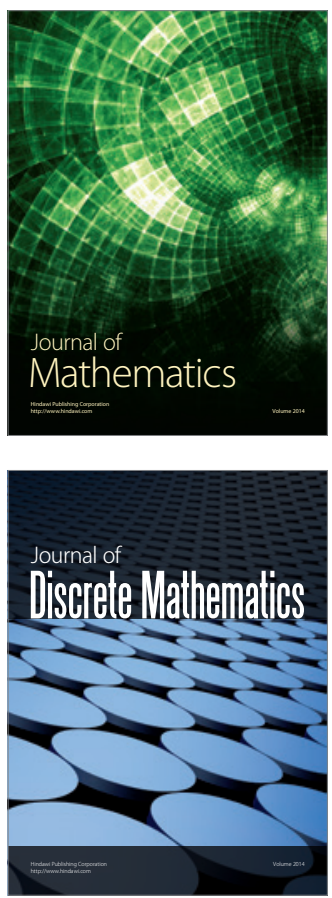

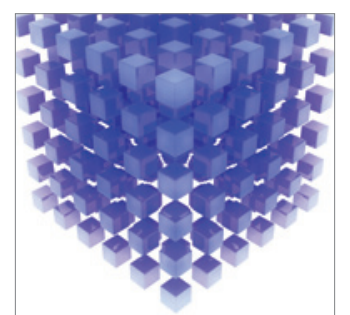

Mathematical Problems in Engineering
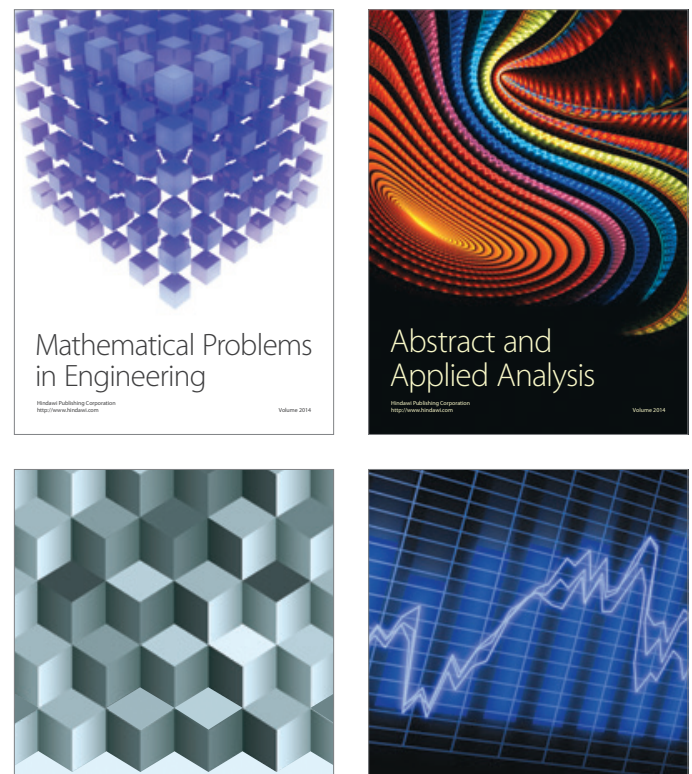

Journal of

Function Spaces

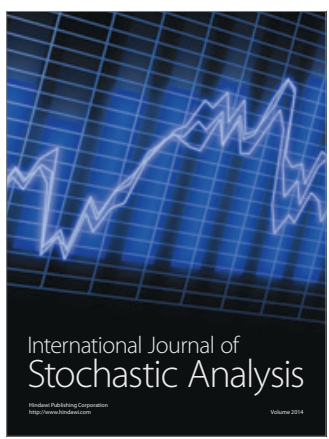

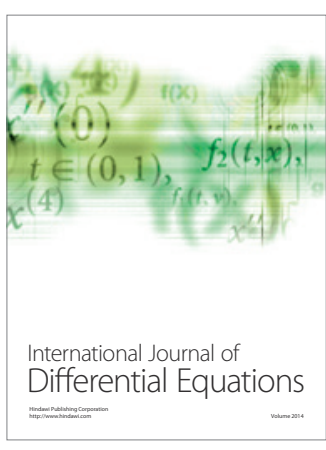
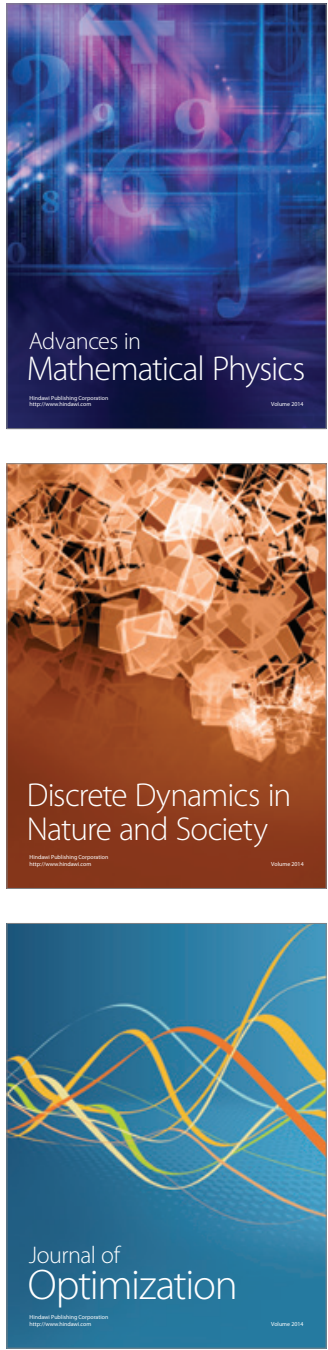